\title{
A precise computation method of Lipschitz Exponent under noisy condition
}

\author{
Xingwei Yan, Wei Wang, Lu Dawei, Zhang Jun, Wan Jianwei \\ School of Electronic Science and Engineering \\ National University of Defense Technology \\ Changsha, China \\ yanxingwei@nudt.edu.cn
}

\begin{abstract}
In noisy condition, the accurate measurement of Lipschitz exponent is very important for many actual applications. All the modulus maxima are utilized to estimate Lipschitz exponent in each maximum line in traditional wavelet transform modulus maxima (WTMM) method, but as the positions and wavelet coefficients are corrupted in some scales by the noise, so the performance of estimation is poor. For this proposed method, the unpolluted modulus maxima are filtrated in each maxima line, and then these modulus maxima and corresponding scales are used to obtain a pre-estimation of Lipschitz exponent. Based on the pre-estimation, different weighted operations are applied to achieve the precise estimation of Lipschitz exponent. Finally, the experimental results verify the good performance of the proposed method.
\end{abstract}

Keywords-Lipschitz Exponent computation, wavelet transform modulus maxima, pre-estimation, noisy condition

\section{INTRODUCTION}

Singularities often carry the most important information in signals. In recent decades, singularity analysis has been applied in many areas. In medical science, the singularities of ECG signals are used to recognize different diseases ${ }^{[1]}$; singularities are also adapted to detect abrupt changes in the vibration signals for fault diagnosis ${ }^{[2]}$; besides, singularities of Echoes present the target localization for through-wall radar $(\mathrm{TWR})^{[3]}$, and it is successfully applied to edges detection $^{[4]}$ and transient signal detection ${ }^{[5]}$.

The Fourier transform has been a tradition main tool for analyzing singularities. Fourier transform is global and provides a description of the overall regularity of signals, but it is not well adapted for finding the location and the spatial distribution of singularities.

Jaffard [6] proposed that the types of singular points could be characterized by Lipschitz exponent in mathematics, and the $n$-dimension signals would be evaluated by $n+1-$ dimension scale space. Thus, Lipschitz exponent is considered be a numerical descriptor to distinguish different types of singularities.

Mallat and Hwang proved in [7] that the wavelet transform could characterize the local singularities of signals, and furthermore, they proposed that the Lipschitz Exponent of a singularity could be estimated by tracing its WTMM curves across scales inside the so-call "cone of influence"(COI). As this method gets high accuracy and low complexity, it has become an important manner for computing Lipschitz exponent of singularities.
However, Mallat's method is based on a particular case of the specifically inequality in [7], and the Lipschitz exponent is obtained as the equal mark is available. There are some modified means for this typical method. The least median of squares regression is applied as the objective function to find Lipschitz exponent in [8]. The asymptote slope of WTMM curve is used to get Lipschitz exponent in [9] and [10]. However, the performance of all mentioned methods declines with the degradation of SNR. Thus, it is imperative to obtain an algorithm to improve the performance of estimation for Lipschitz Exponent under noisy condition, and this paper presents an algorithm to solve this problem.

The rest parts of this paper are organized as follows: In Section II, we present the basic some properties of the continuous wavelet transform, and recall that the local regularity of a signal can be characterized by the decay of its wavelet coefficients across scales. In Section III, a new precise method is proposed to compute Lipschitz exponent of singularities for corrupted signals. In Section IV, a short simulation study is given, and the results demonstrate that the proposed method is more robust and more accurate. Finally Section V contains the conclusions.

\section{CONTINUOUS WAVELET TRANSFORM AND MALLAT'S METHOD}

In order to estimate Lipschit exponent, the Continuous Wavelet Transform and Mallat's method are present as a foundation.

2.1 Continuous Wavelet Transform

The Continuous Wavelet Transform (CWT) is a very useful time-frequency analysis tool with which local features of a signal, such as singularities, can be effectively analyzed. For a signal $f(x) \in \boldsymbol{L}^{2}(\boldsymbol{R})$, the CWT of $f(x)$ is defined as

$$
W f(u, s)=\int_{-\infty}^{+\infty} f(x) \psi_{s}^{*}(x-u) d x=\int_{-\infty}^{+\infty} f(x) \frac{1}{\sqrt{s}} \psi^{*}\left(\frac{x-u}{s}\right) d x
$$

Where $\psi_{s}(x)=(1 / s) \cdot \psi(x / s)$, and $\psi_{s}^{*}(x)$ is the complex conjugate of $\psi_{s}(x)$. The $\psi(x) \in \boldsymbol{L}^{2}(\boldsymbol{R})$ is a mother wavelet satisfying the following admissibility condition:

$$
C_{\psi}=\int_{-\infty}^{+\infty} \frac{|\hat{\psi}(\omega)|^{2}}{|\omega|} d \omega<+\infty
$$

Where $\hat{\psi}(\omega)$ is the Fourier transform of $\psi(x)$. The existence of the integral in (2) requires that: 


$$
\hat{\psi}(0)=0, \quad \text { i.e., } \quad \int_{-\infty}^{+\infty} \psi(x) d x=0
$$

And a wavelet $\psi(\mathrm{x})$ is said to have $n$ vanishing moments if and only if for all positive integers $k<n$, it satisfies

$$
\int_{-\infty}^{+\infty} x^{n} \psi(x) d x=0
$$

The vanishing moment of the wavelet is important for the choice of wavelet for Lipschitz exponent computation, because it provides an upper bound for Lipschitz exponent of singularity characterization. An important property of the CWT is the ability to characterize the local regularity of functions. The local regularity is often measured by the Lipschitz exponent. A popular wavelet using in Lipschitz exponent computation is the $\boldsymbol{n}$-th derivative of the Gaussian function $\theta(t)$ :

$$
\theta_{n}(x)=(-1)^{n} \frac{d^{n} \theta(x)}{d x^{n}}
$$

Where $\theta(x)=\frac{1}{\sqrt{2 \pi}} e^{-\frac{x^{2}}{2}}$.

2.2 Mallat's Method

In order to introduce Mallat's method for Lipschitz exponent estimation, two basic definitions are present firstly. Definition 1 (Lipschitz exponent) A function $f$ is pointwise Lipschitz $\alpha \geq 0$ at $v$, if there exist $K>0$, and a polynomial $p_{v}$ of degree $m=\lfloor\alpha\rfloor$ such that

$$
\forall t \in R, \quad\left|f(t)-p_{v}(t)\right| \leq K|t-v|^{\alpha}
$$

A function $f$ is uniformly Lipschitz $\alpha$ over $[a, b]$ if it satisfies Eq. (6) for all $v \in[a, b]$, with a constant $K$ that is independent of $v$. The Lipschitz exponent of $f$ at $v$ or over $[a$, $b]$ is the sup of $\alpha$ such that $f$ is Lipschitz $\alpha$.

Definition 2 Modulus maxima and Maxima line

- A modulus maxima is defined as a point $\left(u_{0}, s_{0}\right)$ such that for any $u$ in a neighborhood of $u_{0}$ we have $\left|W f\left(u, s_{0}\right)\right|<$ $\left|W f\left(\left(u_{0}, s_{0}\right)\right)\right|$. We still call $\left(u_{0}, s_{0}\right)$ a modulus maxima if the inequality is strict for only the right or only the left side of the neighborhood of $x_{0}$, i.e. if $\left(u_{0}, s_{0}\right)$ is a strict extreme on either the left or the right side of $u_{0}$.

- A maxima line is defined as a connected curve in the scale space $(u, s)$ along all points are modulus maxima.

As definitions of Lipschitz exponent and modulus maxima are present, Theory 1 indicates the relationships between them.

Theory 1 (Jaffard) If $f(t) \in \boldsymbol{L}^{2}(\boldsymbol{R})$ is Lipschitz $\alpha \leq n$ at $v$, then there exists A such that

$$
\forall(u, s) \in \boldsymbol{R}^{+} \times \boldsymbol{R},|W f(u, s)| \leq A s^{\alpha+1 / 2}\left(1+\left|\frac{u-v}{s}\right|^{\alpha}\right)
$$

Conversely, if $\alpha<n$ is not an integer and there exist A and $\alpha^{\prime}<\alpha$ such that

$$
\forall(u, s) \in \boldsymbol{R}^{+} \times \boldsymbol{R},|W f(u, s)| \leq A s^{\alpha+1 / 2}\left(1+\left|\frac{u-v}{s}\right|^{\alpha^{\prime}}\right)
$$

Then $f$ is Lipschitz $\alpha$ at $v$. This theory relates the pointwise regularity of a signal to the decay of its wavelet transform's modulus. However, it can't be used to compute the Lipschitz exponent of singularities conveniently. And the following theory solves this problem

Theorem 2 Let $\psi$ be of compact support, $\boldsymbol{n}$ times continuously differentiable and the $\boldsymbol{n}$-th derivative of a smoothing function. Furthermore let $f$ be a tempered distribution and $u_{0} \in(a, b)$. We assume that $\exists s_{0}>0$ and a constant $C$ such that for $u \in(a, b)$ and $s<s_{0}$, all modulus maxima belong to a cone defined by

$$
|u-v| \leq C s
$$

Then the following statements hold:

- For any $u_{1} \in(a, b), u_{1} \neq u_{0}, f$ is uniformly Lipschitz $n$ in a neighborhood of $u_{1}$.

- Let $\alpha<n, \alpha \notin \mathbf{Z}$, then $f$ is Lipschitz $\alpha$ at $u_{0}$ if and only if there exists a constant $A$ such that at each modulus maxima $(u, s)$ in the cone of equation (9) it holds that

$$
|W f(u, s)| \leq A^{\prime} s^{\alpha+1 / 2}
$$

For the detection of Lipschitz regularity, it is useful to rewrite equation (10) in the following form:

$$
\log |W f(u, s)| \leq \log (A)+(\alpha+1 / 2) \log (s)
$$

For denoting simply, the $A^{\prime}$ is replaced by $A$ in equation (10). Thus, theorem 2 indicates that the Lipschitz regularity at a point $u_{0}$ is given by the maximum slope of straight lines that remain above $\log |W f(u, s)|$ for $(u, s)$ in the cone defined by (9) and on a logarithmic scale.

\section{A NOVEL COMPUTATION METHOD OF LIPSCHITZ EXPONENT}

In this paper, there are all nonoscillating and isolated singularities in the analyzed signal. This section is divided into two parts: the extraction of modulus maximum lines and the computation of Lipschitz Exponent under noisy condition.

3.1 Extraction of modulus maximum lines

Because the position excursion of one modulus maxima often occur at different scales, it is important to choose the corresponding modulus maxima between two neighboring scales. The basic procedure is listed below:

(1) Do dyadic WT by using a quadratic spline of compact support wavelet, and compute the WT coefficients of the input signal. The decomposition level is six;

(2) Record the amplitudes $A_{n}^{j}$, positions $x_{n}^{j}$, and numbers $n$ of WT modulus maxima in all scales $s_{j}$.

(3) In modulus maxima plane, assume $x_{0}$ be the modulus maxima point at scale $s_{j}, x_{1}, x_{2}$ are two neighbor maxima points of $x_{0}$, and $x_{1}^{1}$ is $x_{1}$ corresponding modulus maxima in next scale $s_{j+1}$. Let $W f\left(x_{0}, s_{j}\right)$ be the modulus maxima of $x_{0}$, then the process of determining $W f\left(x_{0}^{1}, s_{j+1}\right)$ is to search an approximation of $W f\left(x_{0}, s_{j}\right)$ in $\max \left(\left(x_{1}, x_{1}^{1}\right), x_{2}\right)$ area at scale $s_{j+1}$ and satisfy $\operatorname{sgn}\left(W f\left(x_{0}^{1}, s_{j+1}\right)\right)=\operatorname{sgn}\left(W f\left(x_{0}, s_{j+1}\right)\right)$;

(4) because the pretend amplitudes and densities of noise are depressed at $1 / \sqrt{2}$ speed as scale accretion, this make the modulus maxima points be controlled mainly by signal and 
some lower value may be pretend amplitudes. Then we can set a threshold as follows:

$$
T_{0}=\frac{\log _{2}(1+2 \sqrt{P})}{J+Z} A
$$

Where $P$ is the presupposed noise power; $J$ is the maximum scale; $Z$ is a constant and generally be 2 of experience value. Then we can delete all the modulus maxima points which are lower than $T_{0}$. Threshold detail coefficients can be applied to the wavelet coefficients for each resolution from 1 to $J$.

(5) If $W f\left(x_{0}^{1}, s_{j+1}\right)$ in $\max \left(\left(x_{1}, x_{1}^{1}\right), x_{2}\right)$ domain satisfies

$$
\frac{W f\left(x_{0}, s_{j}\right)}{W f\left(x_{0}^{1}, s_{j+1}\right)}<T
$$

In which $T=\sqrt{2}$ is the threshold determined by double scale equations, $x_{0}^{1}$ denotes the modulus maxima point.

(6) If $W f\left(x_{0}^{1}, s_{j+1}\right)$ can't be found, then we regard $W f\left(x_{0}, s_{j}\right)$ as the pretend maximum point according Mallat's theory and let $W f\left(x_{0}, s_{j}\right)$ be zero in $x_{0}$ neighboring domain.

(7) Repeat the process above until the wanted scale $s_{J}$.

(8) If all the point pairs $\left(x_{0}, x_{0}^{1}\right),\left(x_{0}^{1}, x_{0}^{2}\right),\left(x_{0}^{2}, x_{0}^{3}\right), \ldots$, exist, they would be the maxima points and be reserved, otherwise they would be deleted. These points $\left(x_{0}, x_{0}^{1}, x_{0}^{2}, x_{0}^{3}, \ldots.\right)$ in corresponding positions in all scales can be linked and known as modulus maxima line.

3.2 A Novel computation method of Lipschitz exponent

According to Mallat' method, the Lipschitz exponent $\alpha$ can be estimated by the use of the slope of linear regression equation obtained by the use of data set $\left\{\log s_{j}, \log \left|W f\left(x_{0}^{j}, s_{j}\right)\right|\right\}$ as equation (12) illustrates.

Step 1 The positions and wavelet coefficients are corrupted in some scales by the noise, so the unpolluted modulus maxima should be filtrated in each maxima line firstly. Especially with smaller scales, the noise part contributes the dominant of the coefficient, so the $s_{j}<$ $\min \left\{s_{J} / 4,10\right\}, W f\left(x_{0}, s_{j}\right)$ is eliminated from data set $\left\{\log s_{j}, \log \left|W f\left(x_{0}^{j}, s_{j}\right)\right|\right\}$. Furthermore, the remained datas are rearranged as $\left\{\log s_{i}, \log \left|W f\left(x_{0}^{i}, s_{i}\right)\right|\right\}, i=0,1, \ldots, m$.

Step2 these remained modulus maxima and corresponding scales are used to obtain a pre-estimation of Lipschitz exponent.

$$
\begin{gathered}
\bar{s}=\frac{1}{m} \sum_{i=0}^{m} \log s_{i}, \quad \bar{a}_{0}=\frac{1}{m} \sum_{i=0}^{m} \log \left|W f\left(x_{0}^{i}, s_{i}\right)\right| \\
\bar{\alpha}=\frac{\sum_{i=0}^{m}\left(\log \left|W f\left(x_{0}^{i}, s_{i}\right)\right|\right)\left(\log s_{i}\right)-m \bar{s} \bar{a}_{0}}{\sum_{i=0}^{m}\left(\log s_{i}\right)^{2}-m \bar{s}^{2}}-\frac{1}{2}
\end{gathered}
$$

Step3 The piecewise function is adapted to different singularities measurement with different pre-estimation of Lipschitz exponent.

$$
\begin{gathered}
\alpha_{i}=\frac{\left|W f\left(x_{0}^{i+1}, s_{i+1}\right)\right|-\left|W f\left(x_{0}^{i}, s_{i}\right)\right|}{s_{i+1}-s_{i}}-\frac{1}{2}, \quad i=0,1, \ldots, m-1 \\
\alpha=\left\{\begin{array}{lc}
\left\{\operatorname{mean} \alpha_{l} \mid \operatorname{mean}\left(\alpha_{l}\right)+2 \operatorname{std}\left(\alpha_{l}\right)>\alpha_{l}>\operatorname{mean}\left(\alpha_{l}\right)\right\}-0.5 & 0<\bar{\alpha}_{0} \leq 0.4 \\
\left\{\operatorname{mean} \alpha_{q} \mid \operatorname{mean}\left(\alpha_{l}\right)+\operatorname{std}\left(\alpha_{l}\right)>\alpha_{l}>\operatorname{mean}\left(\alpha_{l}\right)-\operatorname{std}\left(\alpha_{l}\right)\right\} & -0.50 .4<\bar{\alpha}_{0} \leq 0.7 \\
\left\{\operatorname{mean} \alpha_{q} \mid \operatorname{mean}\left(\alpha_{l}\right)>\alpha_{l}>\operatorname{mean}\left\{\alpha_{l}\right\}-\operatorname{std}\left(\alpha_{l}\right)\right\}-0.5 & 0.7<\bar{\alpha}_{0} \leq 1.0
\end{array}\right.
\end{gathered}
$$

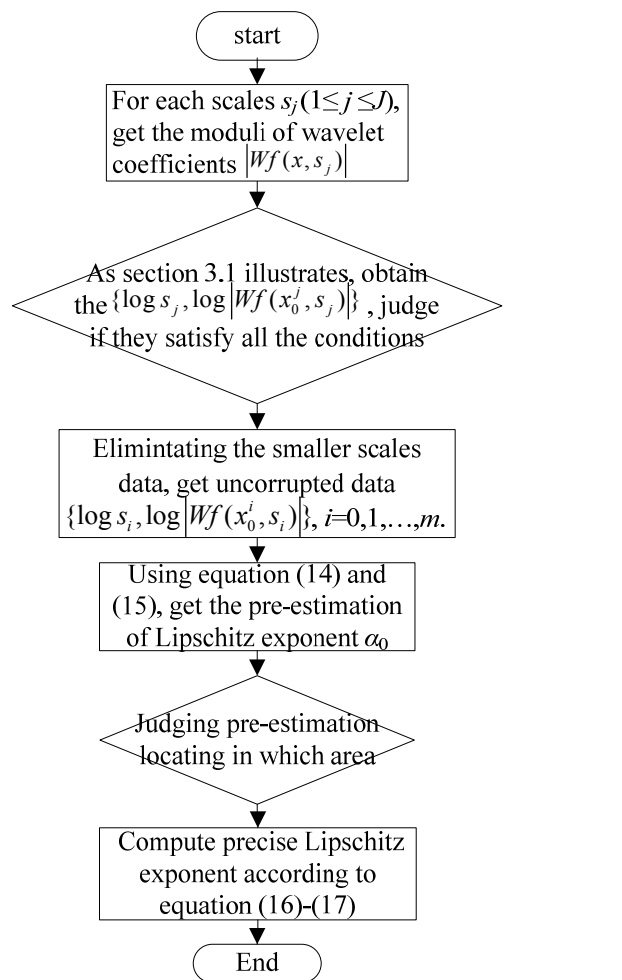

Fig.1 Flowchart of the proposed precise algorithm for Lipschitz exponent

\section{EXPERIMENTS FOR THE LIPSCHITZ EXPONENT EVALUATION}

In this section, we will suppose that $f(x)$ is observed from the white noise condition:

$$
g(x)=f(x)+\sigma \epsilon
$$

In which, $f$ is an unknown function as the measure signal, $\sigma$ is the level of noise and $\epsilon$ are i.i.d. normal distribution with zero mean and variance 1 . We like to analyze the functions (which is considered to the benchmark signal)

$$
f(x)=1-|0.5-x|^{\alpha}
$$

Where $x$ varies from 0 to 1.0 and $\alpha$ is from 0.1 to 0.9 with a 0.1 interval. This function has a Lipschitz exponent $\alpha$. Figure 2 shows this function with $\alpha=0.5$. The computation of Lipschitz exponent $\alpha$ is applied with the proposed method which is contrast with Mallat's method. To estimate $\alpha$ we have a procedure of Eq. (12) and Eq. (15)-(18) in Matlab 7.8.

In this experiment Mexican hat wavelet is used as the mother wavelet, and shown in Fig. 3, satisfying 


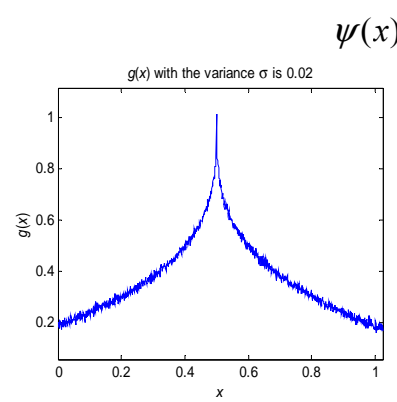

$\psi(x)=\left(1-2 x^{2}\right) e^{x^{2}}$

Fig. 2 The Analysis signal $g(x)$ with $\sigma=0.02$ Fig.3 Mexican hat wavelet $\psi(x)$

According to the proof of Mallat and Hwang [13], if we want to estimate Lipschitz exponents up to maximum value $\boldsymbol{n}$ we require a mother wavelet with at least $\boldsymbol{n}$ vanishing moments. We will estimate $\alpha$ which varies from 0.1 to 0.9 , so Mexican hat wavelet with more than one vanishing moment is a good choice. First we compute the wavelet transform of $g(x)$ and get moduli of the wavelet coefficients as the top of Fig.4. Next step is to find all the modulus maxima in all scales, and to extract the maxima lines as the bottom of Fig.4, while the three red lines indicate the maxima lines. Moreover, it is Fig.5 which presents local details of modulus maximum lines, and the red points display the modulus maximum which dominates by the noise in smaller scales.
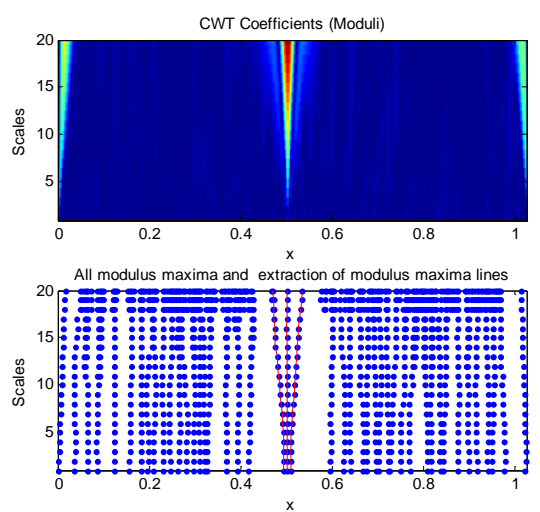

Fig. 4 CWT coefficients, modulus maxima and extraction of maxima lines

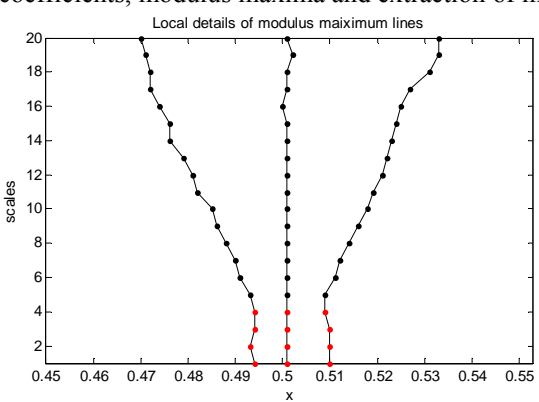

Fig. 5 Local details of modulus maximum lines

The next step is to obtain a log-log plot of scales $s_{j}$ versus coefficient modulus $\left|W f\left(x, s_{j}\right)\right|$, as shown in Fig.6, and the red points are the wavelet coefficient that corrupted by noise in smaller scales. Finally, then to find the slope of corresponding scale and coefficient line using (15), the slope is adapted to get the estimated Lipschitz exponent $\alpha$.

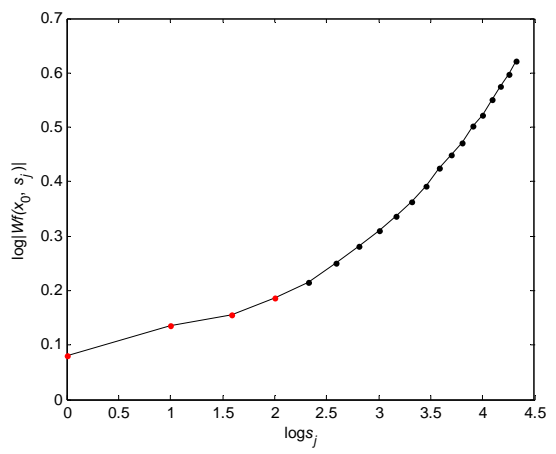

Fig.6 Plot of $\left|\log s_{j}\right|$ vs. $|\log | W f\left(x, s_{j}\right) \mid$

In order to compare my proposed method with Mallat's method, three groups of experiment are made to estimate the Lipschitz exponent of singularities for the analyzed signal $g(x)$. The following three tables are present the results corresponding to the three different types of analyzed signal: the first type of signals $g(x)$ are the pure signal $f(x)$ with no noise for different parameter $\alpha$, and this group of result is the reference group in Table 1; the second type of signals are polluted signals with the noise variance $\sigma=0.03$, and the corresponding $\mathrm{SNR}=20 \mathrm{~dB}$, Table 2 presents the performance of two contrastive methods; while the third group signals are corrupted signals with the noise variance $\sigma=0.05$, and the corresponding $\mathrm{SNR}=12.25 \mathrm{~dB}$, Table 3 gives the performance of two contrastive methods.

Table1 Comparison of Lipschitz exponent estimation for $g(x)$ with no no
\begin{tabular}{|c|c|l|l|l|l|}
\hline \multirow{2}{*}{$\begin{array}{c}\text { True } \\
\alpha\end{array}$} & Mallat's Method & \multicolumn{2}{|c|}{ Proposed Method } & Improve- \\
\cline { 2 - 5 } & $\begin{array}{c}\text { Estima } \\
\text {-tion } \alpha\end{array}$ & $\begin{array}{l}\text { Relative } \\
\text { error }(\%)\end{array}$ & $\begin{array}{l}\text { Estima } \\
\text {-tion } \alpha\end{array}$ & $\begin{array}{l}\text { Relative } \\
\text { error }(\%)\end{array}$ & \\
\hline 0.1 & 0.0601 & $39.90 \%$ & 0.0812 & $18.80 \%$ & $21.10 \%$ \\
\hline 0.2 & 0.1223 & $38.85 \%$ & 0.1704 & $14.80 \%$ & $24.05 \%$ \\
\hline 0.3 & 0.2115 & $29.50 \%$ & 0.2813 & $6.23 \%$ & $23.27 \%$ \\
\hline 0.4 & 0.3488 & $12.80 \%$ & 0.3808 & $4.80 \%$ & $8.00 \%$ \\
\hline 0.5 & 0.4651 & $6.98 \%$ & 0.4936 & $1.28 \%$ & $5.70 \%$ \\
\hline 0.6 & 0.5762 & $3.97 \%$ & 0.5978 & $0.36 \%$ & $3.60 \%$ \\
\hline 0.7 & 0.6843 & $2.24 \%$ & 0.7016 & $0.23 \%$ & $2.01 \%$ \\
\hline 0.8 & 0.7904 & $1.20 \%$ & 0.8021 & $0.26 \%$ & $0.94 \%$ \\
\hline 0.9 & 0.8752 & $2.75 \%$ & 0.9014 & $0.16 \%$ & $2.60 \%$ \\
\hline
\end{tabular}

Table2 Comparison of Lipschitz exponent estimation for corrupted $g(x)$ with $\sigma=0.03$

\begin{tabular}{|c|c|c|c|c|c|}
\hline \multirow{2}{*}{$\begin{array}{c}\text { True } \\
\alpha\end{array}$} & \multicolumn{2}{|c|}{ Mallat's Method } & \multicolumn{2}{|c|}{ Proposed Method } & \multirow{2}{*}{$\begin{array}{l}\text { Improve- } \\
\text { ment (\%) }\end{array}$} \\
\hline & $\begin{array}{l}\text { Estima } \\
\text {-tion } \alpha\end{array}$ & $\begin{array}{l}\text { Relative } \\
\text { error (\%) }\end{array}$ & $\begin{array}{l}\text { Estima } \\
\text {-tion } \alpha \\
\end{array}$ & $\begin{array}{l}\text { Relative } \\
\text { error (\%) }\end{array}$ & \\
\hline 0.1 & 0.0716 & $28.40 \%$ & 0.1261 & $26.10 \%$ & $2.30 \%$ \\
\hline 0.2 & 0.1439 & $28.05 \%$ & 0.2384 & $19.20 \%$ & $8.85 \%$ \\
\hline 0.3 & 0.2174 & $27.53 \%$ & 0.3565 & $18.83 \%$ & $8.70 \%$ \\
\hline 0.4 & 0.3321 & $16.97 \%$ & 0.4240 & $6.00 \%$ & $10.98 \%$ \\
\hline 0.5 & 0.4619 & $7.62 \%$ & 0.5023 & $0.46 \%$ & $7.16 \%$ \\
\hline 0.6 & 0.5223 & $12.95 \%$ & 0.6269 & $4.48 \%$ & $8.47 \%$ \\
\hline 0.7 & 0.6027 & $13.90 \%$ & 0.6723 & $3.96 \%$ & $9.94 \%$ \\
\hline 0.8 & 0.7431 & $7.11 \%$ & 0.7696 & $4.80 \%$ & $2.31 \%$ \\
\hline 0.9 & 0.8536 & $5.16 \%$ & 0.8805 & $2.17 \%$ & $2.99 \%$ \\
\hline
\end{tabular}


Table3 Comparison of Lipschitz exponent estimation for corrupted $g(x)$ with $\sigma=0.05$

\begin{tabular}{|c|l|l|l|l|l|}
\hline \multirow{2}{*}{$\begin{array}{c}\text { True } \\
\alpha\end{array}$} & \multicolumn{2}{|c|}{ Mallat's Method } & \multicolumn{2}{c|}{ Proposed Method } & \multirow{2}{*}{$\begin{array}{l}\text { Improve- } \\
\text { ment (\%) }\end{array}$} \\
\cline { 2 - 5 } & $\begin{array}{c}\text { Estima } \\
\text {-tion } \alpha\end{array}$ & $\begin{array}{l}\text { Relative } \\
\text { error (\%) }\end{array}$ & $\begin{array}{l}\text { Estima } \\
\text {-tion } \alpha\end{array}$ & $\begin{array}{l}\text { Relative } \\
\text { error (\%) }\end{array}$ & \\
\hline 0.1 & 0.0736 & $26.40 \%$ & 0.0798 & $20.20 \%$ & $6.20 \%$ \\
\hline 0.2 & 0.1237 & $38.15 \%$ & 0.1468 & $26.60 \%$ & $11.55 \%$ \\
\hline 0.3 & 0.2575 & $14.17 \%$ & 0.2790 & $7.00 \%$ & $7.16 \%$ \\
\hline 0.4 & 0.3167 & $20.82 \%$ & 0.3796 & $5.10 \%$ & $15.73 \%$ \\
\hline 0.5 & 0.4534 & $9.32 \%$ & 0.5103 & $2.06 \%$ & $7.26 \%$ \\
\hline 0.6 & 0.5237 & $12.72 \%$ & 0.5753 & $4.12 \%$ & $8.60 \%$ \\
\hline 0.7 & 0.6354 & $9.23 \%$ & 0.6774 & $3.23 \%$ & $6.00 \%$ \\
\hline 0.8 & 0.7098 & $11.28 \%$ & 0.7751 & $3.11 \%$ & $8.16 \%$ \\
\hline 0.9 & 0.8479 & $5.79 \%$ & 0.8932 & $0.76 \%$ & $5.03 \%$ \\
\hline
\end{tabular}

Table 1 shows that the Mallat' method gives significant estimation error in the range of Lipschitz exponent $\lambda \leq 0.4$ in which the relative errors are more than $12 \%$ under no noise condition. The Mallat's method obtains better performance for pure signals with Lipschit exponent in the range of 0.5 to 0.9 , resulting in relative error less than $7 \%$. Furthermore, while the values of Lipschitz exponents are in the interval $[0.1,0.4]$, Mallat's method trends to get smaller estimations. However, my proposed method gets better estimation in all the Lipschitz exponent range [0.1, 0.9]. Especially in the range $[0.1,0.4]$, the proposed method achieves great performance improvement. As the Table 2 and Table 3 indicate that the Mallat's method is significant sensitive to noise, it gains worse estimations of Lipschitz exponent under both two noisy conditions. However, the proposed method is less affected by noise, and particularly, it achieves better performance in the range of $(0.5,0.9)$ for Lipschitz exponent estimation while the Mallat's method loses precision in the same range. More concretely, the relative errors are within the scope of $9 \%$ under two noisy condition for Lipschitz exponent in $(0.5,0.9)$, while the Mallat's method gets rather large relative errors under some condition. Unfortunately, all the tables show that measuring singularity of signal through estimating Lipschitz exponent is not reliable in the range of $[0.1,0.3]$ for both two method, though the proposed method achieves comparatively better performance. This is because the estimation is based on an inequality [11] and not on an equation. Besides, the proposed method is also validated to get significant improvement comparison with Mallat's method for other two type of signals $f(x)$, but the results are not present due to the length of paper.

In conclusion, when the noise is present, the Mallat's method suffers a significant effect and gets rather poor performance, while the proposed method has strong robustness and achieves better performance than Mallat's method. The main reason is that the proposed method obtains a pre-estimation for the Lipschitz exponent as if the priori information is gained, and according to the pre- estimation, the corresponding weighted methods are adapted to get a more precise Lipschitz exponent.

\section{CONCLUSION}

The traditional method based on WTMM obtains inaccuracy estimation of Lipschitz exponent under noise condition, and in order to conquer this problem, a new precise algorithm is proposed. The different characters are used for the modulus maxima points between the noise and true signal dominating to eliminate the modulus maxima points of noise. And then, the remaining modulus maxima points and corresponding scales are adapted to get the preestimation of Lipschitz exponent. Based on the preestimation, arbitrary neighboring scales and corresponding modulus maxima point, the final estimation of Lipschitz exponent is obtain by farther analysis. As the modulus maxima points of noise are removed and two steps are applied to compute Lipschitz exponent, the effect of noise can be almost eliminated, so the poposed method can achieve more correct value of Lipschitz exponent. Finally, the experiment results validate the efficiency of the proposed algorithm for Lipschitz exponent estimation under noisy condition.

\section{REFERENCES}

[1] Jalil Bushra, Laligant Olivier, Fauvet Eric, Beya Ouadi. Detection of QRS Complex in ECG signal based on clasiffication approach [C]. Proceedings of 2010 IEEE 17th International Conference on Image Processing, September, 26-29, 2010, Hongkong.

[2] SUN Q. Singularity analysis using continuous wavelet transform for bearing fault diagnosis [J]. Mechanical systems and Signal Processing, 2002, 16(6): 1025-1041..

[3] Chen Xiaoli, Tian Mao, Guo Jing. Singularity Detection of TWR Echoes Based on Correlation[C]. IEEE 10th International Conference on Signal Processing (ICSP 2010), Beijing, China, October 24-28, 2010 ..

[4] S Mallat, S Zhong. Characterization of signals from multiscale edges [J]. IEEE Transaction on Pattern Analysis Machine Intelligence, 1992, 14(7): 710- 732 .

[5] Wang Yang, Sun Tao, Huang Tianshu, Sun Dong. Isolated Singularity points recognition of Hydroelectric generators fault signals based on CWT [C]. IEEE 7th International Conference on Signal Processing(ICSP 2004), Beijing, China, August 31-September 4, 2010.

[6] J S. Jaffard and Y. Meyer. Wavelet Methods for Pointwise Regularity and Local Oscillations of Functions, volume 123. American Mathematical Society, September 1996.

[7] S. Mallat and L. Hwang. Singularity detection and processing with wavelets [J]. IEEE Transactions on Information Theory, 38(2):617642, 1992.

[8] C.L.Tu and W.L.Hwang. Analysis of singularities from modulusm axima of complex wavelets [J].IEEE Trans. Inf. Theory, vol.51, no.3, pp.1049-1062, Mar.2005

[9] J. Zhong and R. Ning. Image Denoising Based on Wavelets andMultifractals for Singularity Detection[J]. IEEE Trans. Image Processing., vol.14, no.10, pp.1435-1447, Oct.20

[10] E.W. Abel, H. Y. Meng, A. Forster and D. Holder. Singularity characteristics ofneedle EMG IP signals [J].IEEE Trans.Biomed.Eng, vol.53, no.2, pp.319-225, Feb.2006 